VOL. 27 (1983), 321-328.

\title{
ENTIRE FUNCTIONS HAVING ASYMPTOTIC FUNCTIONS
}

\author{
P.C. FEnTON
}

It is shown that an entire function having $k$ distinct entire asymptotic functions of order less than $\frac{1}{4}$ is of lower order $\frac{1}{2} k$, mean type at least; further that if $f$ is of lower order $\frac{1}{2} k$, mean type, then its order is $\frac{1}{2} k$.

\section{Introduction}

An entire function $w$ is called an asymptotic function for the entire function $f$ if $f(z)-w(z) \rightarrow 0$ as $z \rightarrow \infty$ along some curve joining 0 to $\infty$, called an asymptotic curve. A consequence of Wiman's theorem is that distinct asymptotic functions of order less than $\frac{1}{2}$ associated with the same function $f$ cannot have the same asymptotic curve, and this together with the Denjoy-Carleman-Ahlfors theorem lends support to the following conjecture, which appears as Problem 2.3 in Hayman's book of problems [4].

If $f$ is an entire fronction having $k$ distinct asymptotic functions of order less than $\frac{1}{2}$ then $f$ has order $\frac{1}{2} k$ at least.

When the asymptotic functions are constants this is a weak version of the Denjoy-Carleman-Ahlfors theorem. In a posthumous paper [7] Somorjai showed that if the orders of the asymptotic functions are less than about $1 / 30$ then the stronger conclusion

$$
\alpha=\underset{r \rightarrow \infty}{\lim \inf } r^{-k / 2} \log M(r, f)>0
$$

Received 9 December 1982. 
holds, where $M(r, f)$ is the maximum modulus of $f$. The proof employs a differential inequality due to Carleman. Denjoy himself had shown earlier that if $f$ is of finite order $\mu$ and all asymptotic curves are half-lines then the number of asymptotic functions of order less than $1 /\left(2+\mu^{-1}\right)$ is no more than $2 \mu$; see [3] for details. In this note we show that the proof of the "regularity" version of the Denjoy-Carleman-Ahlfors theorem due to Heins [5] can be adapted to bear on the foregoing conjecture. We shall prove:

(1.2) If $f$ is an entire finction having $k$ distinct asymptotic functions of order less than $\frac{1}{4}$ then (1.1) holds. Moreover, if $\alpha<\infty$, then $f$ has order $\frac{1}{2} k$.

\section{A lemma}

It is apparent that we may assume that the asymptotic curves are not self-intersecting and also that they are composed of straight line segments, with only finitely many segments between any two of their points. Let us call such curves segmental.

Suppose that $\Gamma_{1}$ and $\Gamma_{2}$ are two segmental curves joining 0 to $\infty$ which are either identical or else intersect in the finite plane only at 0 . In either case the complement of $\Gamma_{1} \cup \Gamma_{2}$ contains an unbounded, simply-connected domain $\Omega$ with $\partial \Omega=\Gamma_{1} \cup \Gamma_{2}$. Given $R>0$ let $\Omega(R)$ be the component of $\Omega \cap \Delta(R)$ which has 0 as a boundary point, and for each $r \in(0, R)$ let $\theta(r)$ be the angular measure of $\Omega(R) \cap C(r)$; here $\Delta(r)$ is the open disc centred at 0 with radius $r$, and $C(r)$ is its boundary. Suppose that $u(z)$ is a subharmonic function in $\Omega(R)$ which satisfies, for each $z \in \partial \Omega(R) \cap\left(\Gamma_{1} \cup \Gamma_{2}\right)$,

$$
\underset{\zeta \rightarrow z}{\lim \sup } u(\zeta) \leq \phi(|z|)
$$

where $\phi$ is increasing and continuously differentiable on $(0, R]$. The upper limit in (2.1) is to be taken as $\zeta$ approaches $z$ from within $\Omega(R)$. We shall show that, for each $r \in(0, R)$, 
(2.2) $\sigma(r, R) \leq \phi(r)+6 \int_{r}^{R} \phi^{\prime}(t) \exp \left(-\pi \int_{r}^{t} d s / s \theta(s)\right) d t$

$$
+6 \sigma(R, R) \exp \left\{-\pi \int_{r}^{R} d s / s \theta(s)\right\},
$$

where

$$
\sigma(t, R)=\sup \{u(z): z \in \bar{\Omega}(R) \cap C(t)\} .
$$

Given $r \in(0, R)$ let $z_{0} \in S_{r}=\Omega(R) \cap C(r)$. For $t \in(r, R)$ let $C_{1}(t)$ be an arc of $S_{t}$ which is not separated from $z_{0}$ by any other arc of $S_{t} \cdot C_{1}(t)$ cuts $\Omega(R)$ into two subdomains, $\Omega_{1}(t)$ containing $z_{0}$, and $\Omega_{1}^{\prime}(t)$. If there is an arc of $S_{t}$ remaining in $\Omega_{1}(t)$ let $C_{2}(t)$ be one which is not separated from $z_{0}$ by any of the others, let $\Omega_{2}(t)$ be the subdomain of $\Omega_{1}(t)$ cut off by $C_{2}(t)$ which contains $z_{0}$ and let $\Omega_{2}^{\prime}(t)$ be the other. And so on. After a finite number $(m=m(t)$ say) of steps we arrive at a domain $\Omega(t)=\Omega_{m}(t) \subseteq \Delta(t), m$ domains $\Omega_{1}^{\prime}(t), \ldots, \Omega_{m}^{\prime}(t)$ and $m$ arcs $C_{1}(t), \ldots, C_{m}(t)$. These domains and arcs are mutually disjoint and their union is $\Omega(R)$. Since $\Omega(t)$ is the unique component of $\Omega(R) \cap \Delta(t)$ - which contains $z_{0}$ we have $\Omega(t) \subseteq \Omega(s)$ for all $r<t \leq s<R$; thus if $\partial_{i}(t)=\{|z|>t\} \cap \partial \Omega_{i}(t)$, $i=1, \ldots, m$, and $\partial(t)=\mathrm{U}_{i}(t)$ then $\partial(t) \supseteq \partial(s)$ for $r<t \leq s<R$.

It is clear that

$$
\omega\left(z_{0}, \partial_{i}(t), \Omega(R)\right) \leq \omega\left(z_{0}, C_{i}(t), \Omega(t)\right)
$$

for $1 \leq i \leq m(t)$, where $\omega$ represents harmonic measure, and so

$$
\omega\left(z_{0}, \partial(t), \Omega(R)\right) \leq 6 \exp \left\{-\pi \int_{p}^{t} d s / s \theta(s)\right\}
$$

from (for example) Kennedy [6, Lema 4]. Given $r^{\prime} \in(r, R)$ and a positive integer $N$ let $t_{j}=r^{\prime}+j\left(R-r^{\prime}\right) / N, 0 \leq j \leq N$, and consider 


$$
\begin{aligned}
h_{N}(z)=\phi\left(r^{\prime}\right)+\sum_{j=1}^{N}\left\{\phi\left(t_{j}\right)-\phi\left(t_{j-1}\right)\right\} \omega(z, & \left.\partial\left(t_{j-1}\right), \Omega(R)\right) \\
& +\sigma(R, R) \omega(z, \partial \Omega(R) \cap C(R), \Omega(R)) .
\end{aligned}
$$

$h_{N}$ is harmonic in $\Omega(R)$. Moreover if $z \in \partial \Omega(R)$ then $h_{N}(z) \geq \underset{\zeta \rightarrow z}{\lim \sup } u(\zeta)$. This is clear from the hypotheses of (1.2) if $|z| \leq r^{\prime}$ or $|z|=R$, while otherwise $z \in \partial\left(t_{i-1}\right)-\partial\left(t_{i}\right)$ for exactly one $i$ and therefore

$$
h_{N}(z) \geq \phi\left(r^{\prime}\right)+\sum_{j=1}^{i}\left(\phi\left(t_{j}\right)-\phi\left(t_{j-1}\right)\right)=\phi\left(t_{i}\right) \geq \phi(|z|) .
$$

Allowing $N \rightarrow \infty, r^{\prime} \rightarrow r$, after taking account of (2.3) we deduce (2.2).

\section{Proof of the result}

The first concern is to show that any two of the asymptotic curves are ultimately non-intersecting. Suppose this is not the case, that $\gamma_{1}$ and $\gamma_{2}$ are two asymptotic curves with points of intersection $z_{n} \rightarrow \infty \cdot \gamma_{1}$ and $\gamma_{2}$ determine a sequence of bounded domains $D_{n}$, the union of the boundaries of which is $\gamma_{1} \cup \gamma_{2}$. Let $w_{1}$ and $w_{2}$ be the asymptotic functions associated with $\gamma_{1}$ and $\gamma_{2}$ and let $W(z)=w_{1}(z)-w_{2}(z)$. Since $\gamma_{1}$ and $\gamma_{2}$ intersect at $z_{n} \rightarrow \infty, W$ is not constant. Let

$$
v(z)=\log \left|\left[f(z)-\frac{1}{2}\left(w_{1}(z)+w_{2}(z)\right)\right]^{2}-\frac{1}{4} W(z)^{2}\right|,
$$

so that $v(z)$ is subharmonic. Under the hypotheses of (1.2) there is a constant $K_{1}>0$ such that $v(z) \leq \log ^{+}|W(z)|+K_{1}$ for all $z$ on $\gamma_{1}$ and $\gamma_{2}$. We apply (2.2) with $\Gamma_{1}=\Gamma_{2}=\gamma_{1}, u(z)=u_{R}(z)-$ the harmonic function in $\Omega(R)$ with boundary values $\log ^{+}|W(z)|$ - and $\phi(t)=t^{\rho+\varepsilon}+K_{2}$, where $\rho<\frac{1}{4}$ is the order of $W$, and $\varepsilon$ and $K_{2}$ are positive with $\rho+\varepsilon<\frac{2}{2}$. This gives

$$
u_{R}(z) \leq 6 \sqrt{|z| / R} \log ^{+} M(R, W)+\frac{1+10(\rho+\varepsilon)}{1-2(\rho+\varepsilon)}|z|^{\rho+\varepsilon},
$$


using $\theta(s) \leq 2 \pi$. Since, for each fixed $\underline{n}, D_{n} \subseteq \Omega(R)$ for all large $R$ we deduce that

$$
v(z) \leq \frac{1+10(\rho+\varepsilon)}{1-2(\rho+\varepsilon)}|z|^{\rho+\varepsilon}+O(1)
$$

for all $z$ in $U D_{n}$. It follows that the subharmonic function

$$
V(z)= \begin{cases}\log ^{+}|W(z)|+K_{1} & , z \notin U D_{n}, \\ \max \left\{\log ^{+}|W(z)|+K_{1}, v(z)\right\}, & z \in U D_{n},\end{cases}
$$

has order $\rho$ at most. Now Denjoy [2] has shown that if $u$ is subharmonic of order $\mu \in[0,1)$ and

$$
A(r, u)=\inf \{u(z):|z|=r\}, B(r, u)=\max \{u(z):|z|=r\},
$$

then, given $\mu^{\prime} \in(\mu, 1)$,

$$
\int_{x}^{\infty}\left\{A(r, u)-\cos \pi \mu^{\prime} B(r, u)\right\} r^{-\mu^{\prime}-1} d r>K\left(\mu^{\prime}\right) x^{-\mu^{\prime}} B(x, u)
$$

for all $x>0$. Here $K\left(\mu^{\prime}\right)$ is a positive constant. Since every circle about the origin intersects $\gamma_{1}$ and $\gamma_{2}$ we have $A(r, V) \leq \log ^{+} M(r, W)+K_{1}$ for all $r$. Thus if $\rho^{\prime} \in\left(\rho, \frac{1}{4}\right)$ then

$$
\int_{x}^{\infty}\left\{\log m(r, W)-\cos ^{2} \pi \rho^{\prime} B(r, V)\right\} r^{-\rho^{\prime}-1} d r>0
$$

for all large $x$, where $m(r, W)$ is the minimum modulus of $W$. It follows that, for a sequence $R_{n} \rightarrow \infty$,

$$
B\left(R_{n}, V\right)<\sec ^{2} \pi \rho^{\prime} \log m\left(R_{n}, W\right)<(2-n) \log m\left(R_{n}, W\right)
$$

for some $n>0$, since $\rho^{\prime}<\frac{1}{4}$. The circle $|z|=R_{n}$ intersects one of the domains, $D_{N}$ say, formed by $\gamma_{1}$ and $\gamma_{2}$ and so there is an arc of it in $D_{N}, \gamma$ say, joining a point of $\gamma_{1}$ to a point of $\gamma_{2}$. Taking account of the definition of $V$ we deduce that since $W$ is uniformly large on $\gamma$ either

$$
f(z)-\frac{1}{2}\left(w_{1}(z)+w_{2}(z)\right)=W(z)\left(\frac{1}{2}+o(1)\right) \quad(z \in \gamma)
$$


or the same except for a minus sign on the right. In either case a contradiction arises - in the case of (3.1) for instance take $z$ to be the end-point of the arc on $\gamma_{2}$. We conclude that the asymptotic curves are ultimately non-intersecting and therefore may be altered near the origin so as to be intersecting only at 0 . For the remainder of the proof we suppose this done.

Let $w_{1}, \ldots, w_{k}$ be the asymptotic functions with corresponding asymptotic curves $\gamma_{1}, \ldots, \gamma_{k}$ in clockwise order, let $\Omega_{i}$ be the unbounded simply-connected domain between $\gamma_{i}$ and $\gamma_{i+1}\left(\gamma_{k+1} \equiv \gamma_{1}\right)$ and let $\theta_{i}(t)$ be the angular measure of $\Omega_{i} n C(t)$. Let

$$
\begin{aligned}
w_{i}(z)=w_{i}(z)-w_{i+1}(z) \quad\left(w_{k+1} \equiv w_{1}\right) \text {, let } \\
v_{i}(z)=\log \left|\left[f(z)-\frac{1}{2}\left(w_{i}(z)+w_{i+1}(z)\right)\right]^{2}-\frac{1}{4} W_{i}(z)^{2}\right|
\end{aligned}
$$

and let

$$
\sigma_{i}(t)=\max \left\{v_{i}(z): z \in \Omega_{i} \cap C(t)\right\} .
$$

For each $i$ there is a constant $K_{i}>0$ such that $v_{i}(z) \leq \log ^{+}\left|W_{i}(z)\right|+K_{i}$ on $\gamma_{i}$ and $\gamma_{i+1}$. We apply (2.2) with $\Gamma_{1}=\gamma_{i}, \Gamma_{2}=\gamma_{i+1}, \quad \Omega=\Omega_{i}$ and $u(z)$ the harmonic function in $\Omega(R)$ with boundary values $\log ^{+}\left|W_{i}(z)\right|$ on $\Gamma_{1}$ and $\Gamma_{2}$ and $\sigma_{i}(R)$ on $\partial \Omega(R) \cap C(R)$. With $\phi$ as above we deduce that, for all large $R$,

$$
\sigma_{i}(r)<6 \sigma_{i}(R) \exp \left\{-\pi \int_{r}^{R} d s / s \theta_{i}(s)\right\}+o\left(r^{\rho+\varepsilon}\right) .
$$

It follows that $\lim$ inf $R^{-\frac{1}{2}} \sigma_{i}(R)>0$. For if this were not so the first term on the right of (3.2) would tend to zero as $R \rightarrow \infty$ through a sequence of values and we should thus arrive at $\sigma_{i}(r)=O\left(r^{\rho+\varepsilon}\right)$ for each $\varepsilon>0$; hence

$$
V_{i}(z)= \begin{cases}\log ^{+}\left|W_{i}(z)\right|+K_{i} & , z k \Omega_{i}, \\ \max \left\{\log ^{+}\left|W_{i}(z)\right|+K_{i}, v_{i}(z)\right\}, & z \in \Omega_{i},\end{cases}
$$


would have order $\rho$ and just as before this leads to a contradiction when $\rho<\frac{1}{4}$, provided $W_{i}$ is not constant. If $W_{i}$ is constant then the earlier argument is inapplicable; however, it is easy to see that $v_{i}$ is bounded in $\Omega_{i}$ (take $\phi$ constant in (2.2)). But then $f(z)-w_{i}(z)$ is bounded in $\Omega_{i}$ and has distinct limits on $\gamma_{i}$ and $\gamma_{i+1}$, which violates the Phragmén-Lindelöf principle [1, p. 3] and again we arrive at a contradiction. We rearrange (3.2) to give

$$
(I+o(I)) \sigma_{i}(r) \leq \sigma_{i}(R) \exp \left\{-\pi \int_{r}^{R} d s / s \theta_{i}(s)\right\} \text {. }
$$

This is the equivalent of Heins' (2.2) and (3.2) [5]. The remainder of the proof simply reproduces Heins' arguments and we content ourselves to demonstrate this only so far as proving (1.1), which can be quickly done. As we have noticed $\sigma_{i}(r)>0$ for all large $r$ and so, from $(3.3)$,

$$
(1+o(1)) \prod_{1}^{k} \sigma_{i}(r) \leq \exp \left\{-\pi \int_{r}^{R}\left[\sum_{1}^{k} \frac{1}{\theta_{i}(s)}\right] \frac{d s}{s}\right\} \prod_{1}^{k} \sigma_{i}(R) \text {. }
$$

Applying Hölder's inequality to the identity $k=\sum_{1}^{k} \theta_{i}^{\frac{1}{2}} \theta_{i}^{-\frac{1}{2}}$ we obtain $\sum_{1}^{k} \theta_{i}^{-1} \geq k^{2} / 2 \pi$ and hence

$$
(1+o(1)) r^{-k^{2} / 2} \prod_{1}^{k} \sigma_{i}(r) \leq R^{-k^{2} / 2} \prod_{1}^{k} \sigma_{i}(R)
$$

From this it follows that $\lim$ inf $\left\{R^{-k^{2} / 2} \prod_{1}^{k} \sigma_{i}(R)\right\}>0$ which implies $(1.1)$

\section{References}

[1] M.L. Cartwright, Integral functions (Cambridge Tracts in Mathematics and Mathematical Physics, 44. University of Cambridge Press, Cambridge, 1956). 
[2] Arnaud Denjoy, "Sur un théorème the Wiman", C.R. Acad. Sci. Paris 193 $(1931), 828-830$.

[3] Arnaud Denjoy, "L'allure asymptotique des fonctions entières d'ordre fini", C.R. Acad. Sci. Paris 242 (1956), 213-218.

[4] W.K. Hayman, Research problems in function theory (Athlone Press [University of London], London, 1967).

[5] Maurice Heins, "On the Denjoy-Carleman-Ahlfors theorem", Ann. of Math. 49 (1948), 533-537.

[6] P.B. Kennedy, "On a conjecture of Heins", Proc. London Math. Soc. (3) 5 (1955), 22-47.

[7] Gábor Somorjai, "On asymptotic functions", J. London Math. Soc. (2) 21 (1980), 297-303.

Department of Mathematics, University of Otago,

Dunedin,

New Zealand. 